\title{
HARMONIC CURRENTS IN AN ARRAY OF VARIABLE PHASED POWER SUPPLIES
}

\section{BOOSTER TECHNICAL NOTE \\ NO. 230}

\author{
M. METH and J. SANDBERG
}

July 9, 1998

ALTERNATING GRADIENT SYNCHROTRON DEPARTMENT BROOKHAVEN NATIONAL LABORATORY

UPTON, NEW YORK 11973 


\section{HARMONIC CURRENTS IN AN ARRAY OF VARIABLE PHASED POWER SUPPLIES}

The literature and discussion of an array of multi-phase power supplies generally assumes that the various power supplies are operated in phase (ie. each supply is at maximum voltage and firing angles are equal). In this case the generated harmonic currents completely reinforce, which is not true at the BNL Accelerator installations. Power supplies are independently adjusted to arbitrary voltages by varying the firing angle of the supplies. The phase of the line currents reflect the firing angles of the power supplies. Since the harmonic components of the line currents are typically not in phase, they can either reinforce or cancel. This study shows that in the general case where firing angles are dissimilar, the percent harmonics are always reduced from those generated when identical firing angles are assumed.

Recent measurements of the harmonic composition of line currents at the 480 -volt level and the $13.8 \mathrm{kV}$ level at BNL seem to verify the independent phasing of the various supplies. The arithmetic addition of the various harmonics on the 480-volt line was significantly greater than the composite harmonics on the $13.8 \mathrm{kV}$ feeder, including corrections for the voltage transformation ratio and commutation overlap.

The elements of this phenomenon can be readily explained by examining two-independent power supplies energized from a common feed, see Figure 1. Each power supply is a delta, 6-phase star. Power supply 1 is full on; supply 2 is delayed in phase $30^{\circ}$ (on a 60-hz scale) and has an output of .866 of its maximum voltage. The analysis is on a per unit basis using the rated DC voltages and current as base values. Figure $2 \mathrm{~A}$ is the idealized line current for power supply 1 . The line current for power supply 2 is the same as for 1 but delayed $30^{\circ}(60 \mathrm{~Hz}$ scale). The composite line current is given in Figure 2C. It is obvious that the waveform of Figure $2 \mathrm{~A}$ is richer in harmonics than is the waveform of Figure $2 \mathrm{C}$. Idealized waveforms neglect the rounding of the edges due to commutation overlap. For each of the waveforms analyzed the origin is selected to maintain odd-function symmetry. For example, in Figure $2 \mathrm{C}$ the origin has been translated $15^{\circ}$. For a development of how the parameter of Figure 1 are derived, see Appendix 1.

The harmonic analysis of the current waveforms of Figure $2 \mathrm{~A}$ and Figure $2 \mathrm{C}$ is summarized in Table I. (See Appendix 2) Note the substantial reduction in the 5th and 7th harmonic components (factor of 4) and the reduction in the total harmonic distortion (factor of 2) by the addition of these two waveforms.

The addition of two current waveforms of equal magnitude with a phase difference of $36^{\circ}$ (relative rectified voltage of value 1.0 and 0.81 ) will cancel the 5 th harmonic distortion and reduce the 7 th harmonic distortion by a factor of 1.6 . The 5 th harmonic component will be reduced from this phenomenon for all phase difference that are less than $72^{\circ}$ (rectified voltages of 1.0 and 0.31 ).

As another example of the reduction of harmonic distortion due to the addition of line currents is the case of $45^{\circ}$ phase back. One supply is full-on and the other is phased back $45^{\circ}$. The rectified voltages are 1.0 and 0.707 , the two rectified currents are equal. The harmonic analysis for this case is included and is summarized in Table II. Note that the 5 th, 11th, and 13th harmonic distortion is reduced by a factor of 2.6; where as the 7 th harmonic distortion remains the same. Table 
II summarizes the performance of two 6-pulse supplies as a function of phase-back ( $\alpha$ ) of one of the two supplies.

Harmonic reduction and or cancellation does not affect the power flow between the line and the supply. Power flow requires that the current and voltage be of the same frequency and in phase for real power to flow or in quadrature for reactive power to flow. The integral involving the product of current and voltage of different frequencies, including harmonic relationships, is zero and does not contribute to power flow and to power balance.

The analysis can be performed either in the time domain or in the frequency domain. Time domain analysis involves developing the Fourier Series of the composite waveform. Frequency domain analysis involves linear superposition of the phase - shifted harmonic terms of the individual supplies (See Appendix 2).

The current harmonic distortion from multi-phase power supplies follows the $1 / \mathrm{n}$ rule $(\mathrm{n}=$ harmonic numbers: $5,7,11,13 \ldots$ ) at the lowest voltage level, generally 480 volts at BNL. At distribution and transmission voltage levels $(13.8 \mathrm{kV}$ and $69 \mathrm{kV})$ there is no obvious rule to predicate the value of harmonic currents knowing the currents of the individual power supplies. However, it is obvious from the examples cited that the composite harmonic distortion is significantly less than the arithmetic sum of the individual supplies.

As a second example consider three independent power supplies powered by a common power line. One supply is full on; the other two are phased back; One by $18^{\circ}$ and the other by $36^{\circ}$. Relative rectified voltages are 1.0, 0.951 and 0.809 . The load currents of the three supplies are equal, normalized value of 1.0. Figure $3 \mathrm{~B}$ depicts the composite line current. The harmonic analysis is summarized in Table III and is compared to the composite line current resulting from three independent supplies that are each operated full on.

The line current for the three phased back supplies has considerably less harmonic distortion than the line current for two phased back supplies. In fact, as the number of supplies with random phase back increase, the harmonic distortion on the feeder decreases.

Two conclusions can be drawn from this study: first, harmonic currents at the $13.8 \mathrm{kV}$ and 69 $\mathrm{kV}$ levels must be measured and not calculated from measurements taken at the 480 volt level. Second, measurements of 480 volt harmonic currents made by outside consultants in prior years is not particularly useful in determining the labs harmonic load. This loading is required for the design of power line filters. The employment of the arithmetic sum for the composite current could result in substantial over rating the components of the filter. 


\begin{tabular}{|l|l|l|}
\hline \multicolumn{1}{|c|}{ PARAMETERS } & \multicolumn{1}{|c|}{ Waveform of FIG. 2A } & Waveform of FIG. 2C \\
\hline Fundamental Components, rms & 0.7797 & 1.506 \\
\hline 5th Harmonic rms & 0.1559 & 0.0807 \\
\hline 5th Harmonic \% & $20 \%$ & $5.35 \%$ \\
\hline 7th Harmonic rms & 0.1114 & 0.0576 \\
7th Harmonic \% & $14.3 \%$ & $3.82 \%$ \\
\hline 11th Harmonic rms & 0.0709 & 0.1369 \\
\hline 11 th Harmonic $\%$ & $9.1 \%$ & $9.1 \%$ \\
\hline 13 th Harmonic rms & 0.0599 & 0.1159 \\
\hline 13th Harmonic \% & $7.7 \%$ & $7.7 \%$ \\
\hline RMS Value & 0.8165 & 1.5275 \\
\hline Total Harmonic Distortion rms & 0.2424 & 0.2554 \\
Total Harmonic Distortion \% & $31.1 \%$ & $16.96 \%$ \\
\hline
\end{tabular}

Table I

Harmonic Analysis of Current Waveforms 


\begin{tabular}{|c|c|c|c|c|c|c|c|}
\hline \multirow[b]{2}{*}{ PARAMETERS } & \multicolumn{7}{|c|}{ PHASE BACK } \\
\hline & $0^{\circ}$ & $30^{\circ}$ & $36^{\circ}$ & $45^{\circ}$ & $60^{\circ}$ & $72^{\circ}$ & $90^{\circ}$ \\
\hline Rectified Voltages & $1.0,1.0$ & $1.0,0.867$ & $1.0,0.809$ & $1.0,0.707$ & $1.0,0.5$ & $1.0,0.31$ & $1.0,0$ \\
\hline Displacement Power Factor & 1.0 & 0.966 & 0.951 & 0.924 & 0.866 & 0.809 & 0.707 \\
\hline Fundamental Current rms & 1.559 & 1.507 & 1.483 & 1.441 & 1.350 & 1.262 & 1.103 \\
\hline 5th Harmonic rms & 0.312 & 0.0806 & 0 & 0.119 & 0.270 & 0.312 & 0.2205 \\
\hline Distortion & $20 \%$ & $5.35 \%$ & 0 & $8.3 \%$ & $20 \%$ & $24.7 \%$ & $20 \%$ \\
\hline 7th Harmonic rms & 0.223 & 0.0576 & 0.131 & 0.206 & 0.193 & 0.069 & 0.1575 \\
\hline Distortion & $14.3 \%$ & $3.82 \%$ & $8.83 \%$ & $14.3 \%$ & $14.3 \%$ & $5.45 \%$ & $14.3 \%$ \\
\hline 11th Harmonic rms & 0.142 & 0.137 & 0.135 & 0.054 & 0.123 & 0.115 & 0.1002 \\
\hline Distortion & $9.1 \%$ & $9.1 \%$ & $9.1 \%$ & $3.76 \%$ & $9.1 \%$ & $9.1 \%$ & $9.1 \%$ \\
\hline 13th Harmonic rms & 0.120 & 0.116 & 0.0705 & 0.046 & 0.104 & 0.036 & 0.0848 \\
\hline Distortion & $7.7 \%$ & $7.7 \%$ & $4.75 \%$ & $3.2 \%$ & $7.7 \%$ & $2.9 \%$ & $7.7 \%$ \\
\hline RMS Value & 1.6330 & 1.5275 & 1.506 & 1.472 & 1.414 & 1.3165 & 1.1547 \\
\hline Total Harmonic Distortion rms & 0.486 & 0.246 & 0.2596 & 0.300 & 0.421 & 0.375 & 0.3416 \\
\hline Total Harmonic Distortion \% & $31.1 \%$ & $16.2 \%$ & $17.5 \%$ & $20.8 \%$ & $31.1 \%$ & $29.7 \%$ & $31.1 \%$ \\
\hline
\end{tabular}

Table II

Performance of two 6-pulse supplies as a function of phase-back

$(\alpha)$ of second supply. First supply is not phased-back. 


\begin{tabular}{|c|c|c|}
\hline \multirow[b]{2}{*}{ PARAMETER } & \multicolumn{2}{|c|}{ PHASE BACK } \\
\hline & $0^{\circ}, 0^{\circ}, 0^{\circ}$ & $0^{\circ}, 18^{\circ}, 36^{\circ}$ \\
\hline Rectified Voltage & $1.0,1.0,1.0$ & $1.0,0.951,0.809$ \\
\hline Displacement Power Factor & 1.0 & 0.951 \\
\hline Fundamental Current rms & 2.339 & 2.263 \\
\hline 5th Harmonic rms & 0.468 & 0.156 \\
\hline Distortion & $20 \%$ & $6.9 \%$ \\
\hline 7th Harmonic rms & 0.335 & 0.02 \\
\hline Distortion & $14.3 \%$ & $0.9 \%$ \\
\hline 11th Harmonic rms & 0.213 & 0.064 \\
\hline Distortion & $9.1 \%$ & $2.75 \%$ \\
\hline 13th Harmonic rms & 0.180 & 0.011 \\
\hline Distortion & $7.7 \%$ & $0.5 \%$ \\
\hline 17th Harmonic rms & 0.138 & 0.100 \\
\hline Distortion & $5.9 \%$ & $4.4 \%$ \\
\hline 19th Harmonic rms & 0.123 & 0.119 \\
\hline Distortion & $5.3 \%$ & $5.3 \%$ \\
\hline RMS Value & 2.449 & 2.2804 \\
\hline Total Harmonic Distortion rms & 0.727 & 0.281 \\
\hline Total Harmonic Distortion \% & $31.1 \%$ & $12.43 \%$ \\
\hline
\end{tabular}

Table III

Performance of three 6-pulse supplies. 


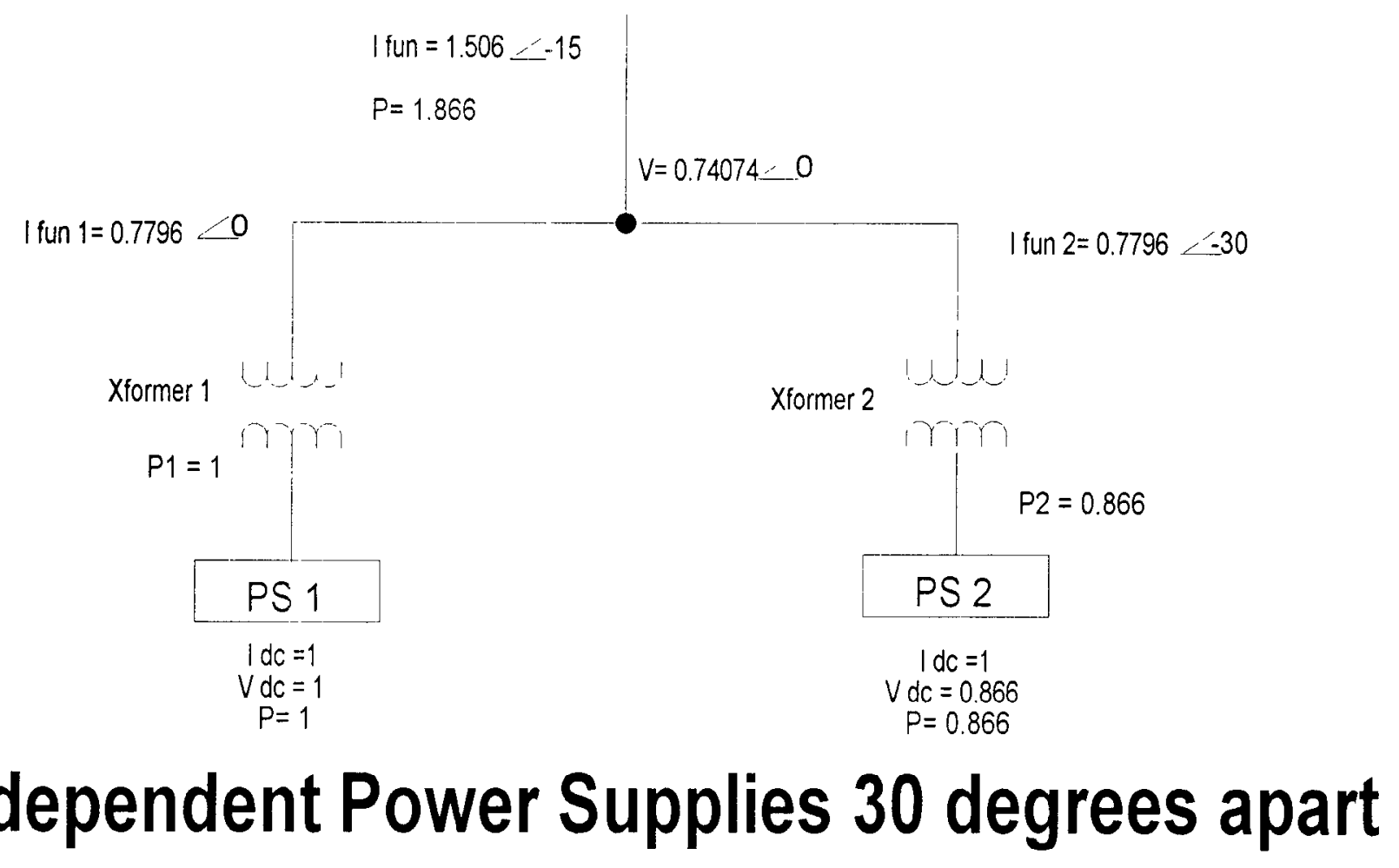

Figure $1 \mathrm{~A}$
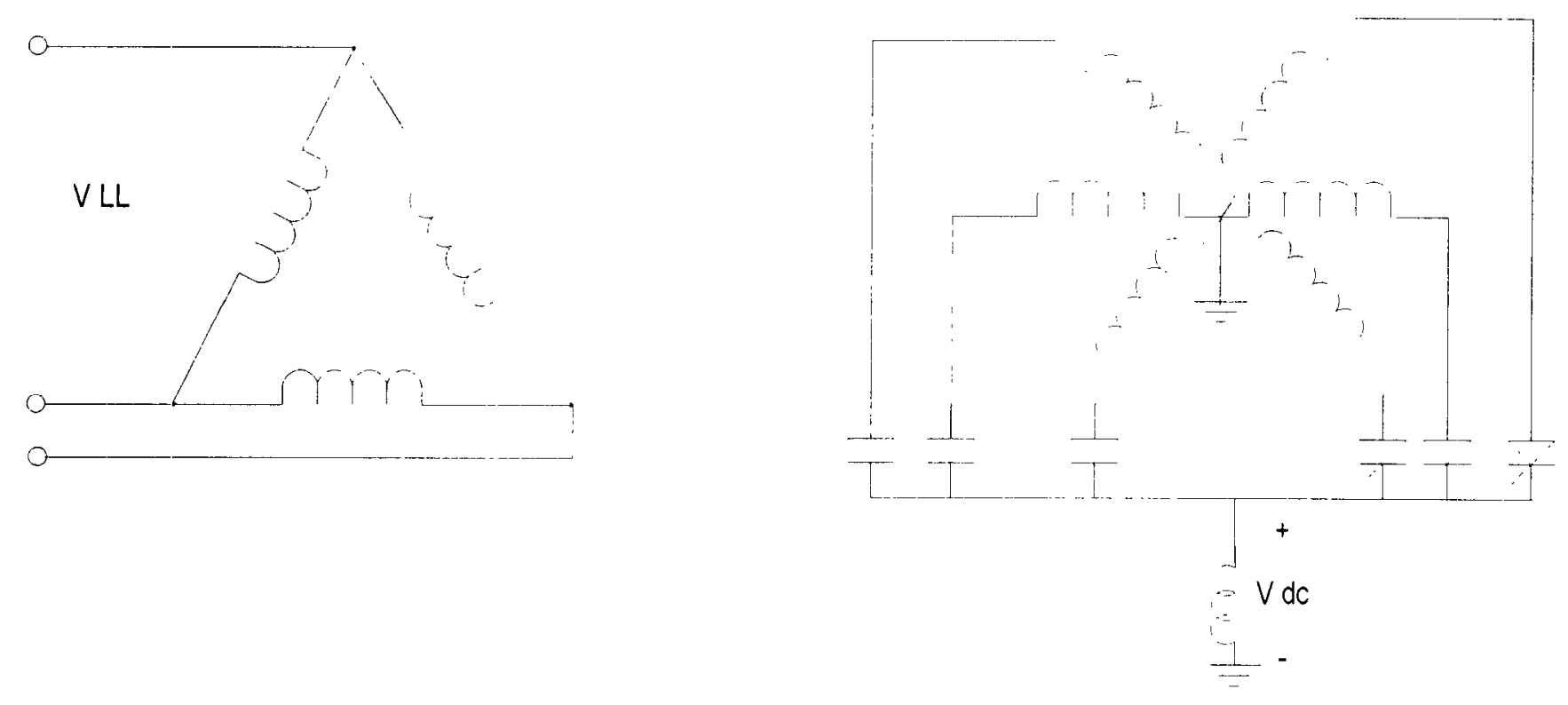

\section{Power Supply; Delta, 6-Phase Star}

Figure $1 \mathrm{~B}$

Figure 1 


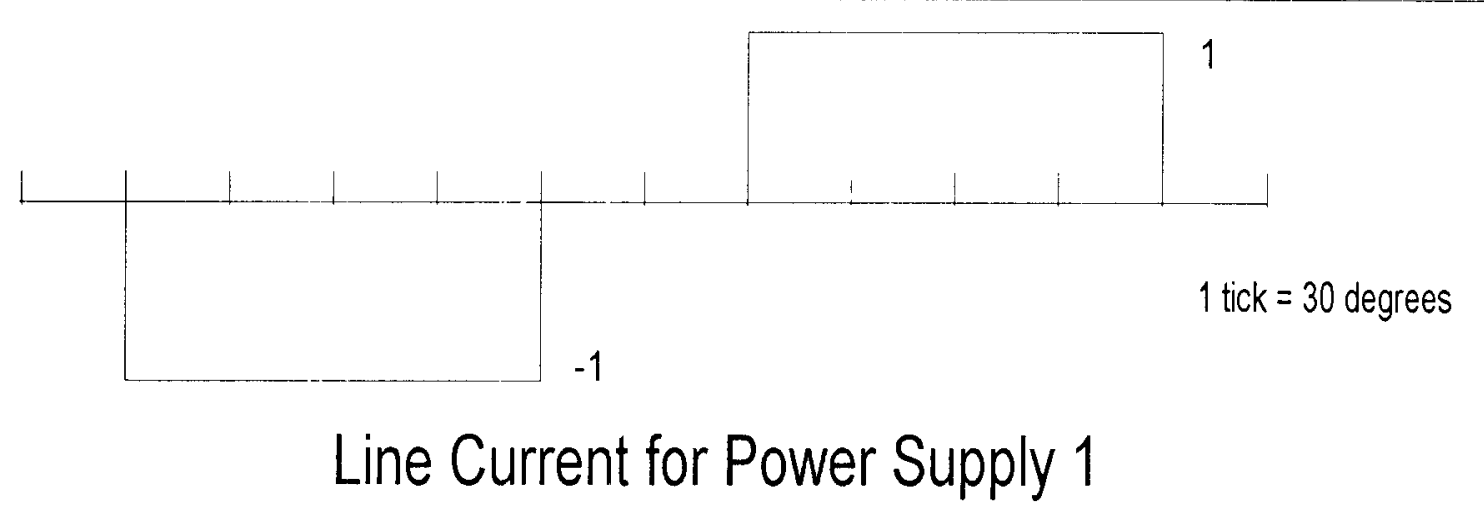

Figure $2 \mathrm{~A}$

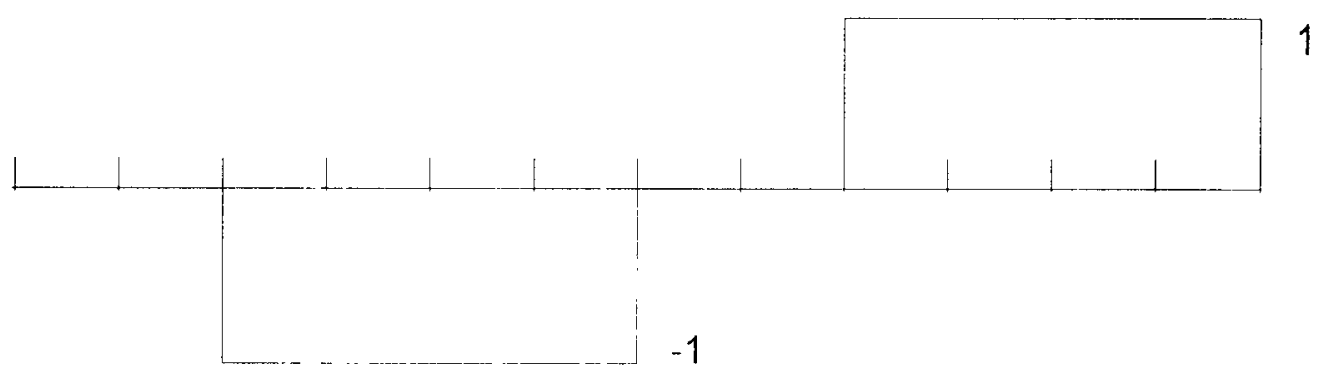

Line Current for Power Supply 2

Figure 2 $\mathrm{B}$

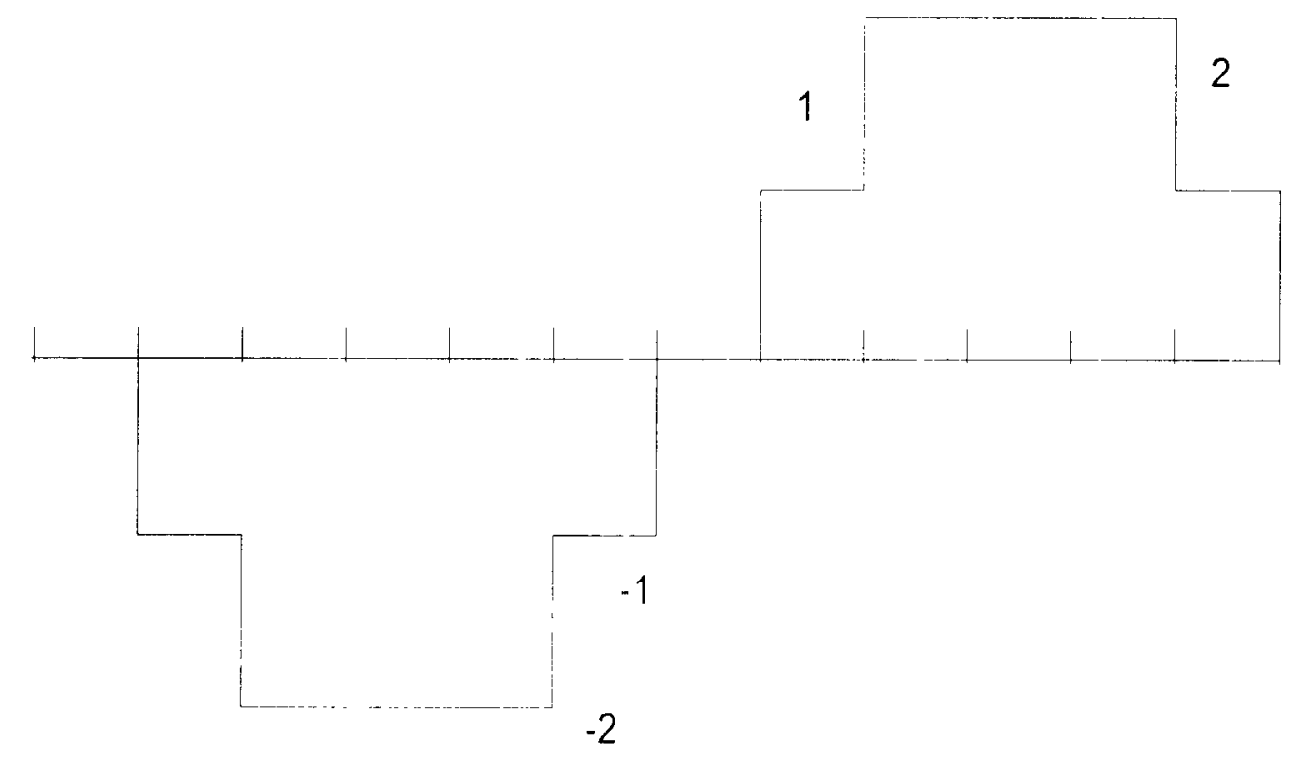

Composite Line Current for Power Supplies $1 \& 2$

Figure $2 \mathrm{C}$

Figure 2

$-7-$ 
1
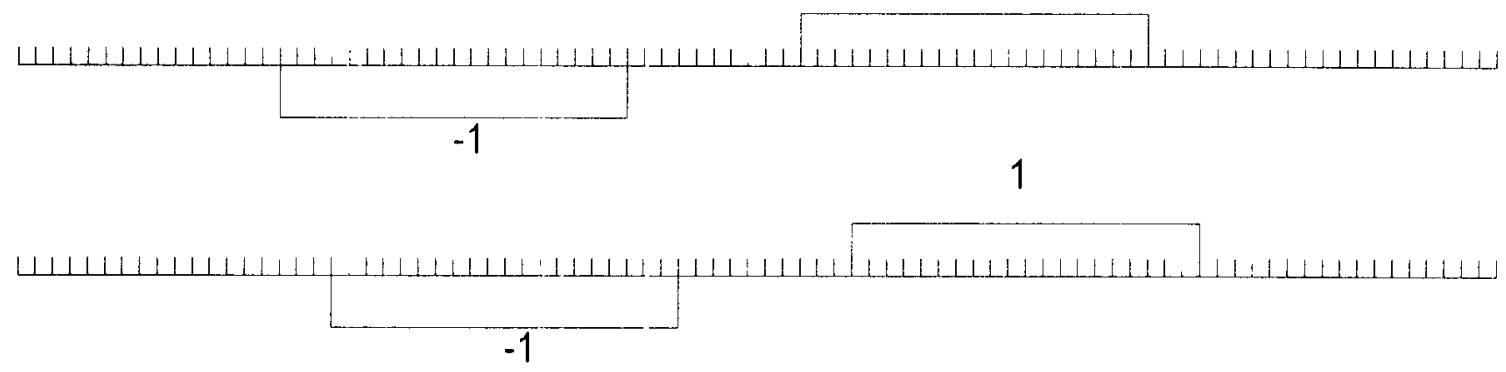

1

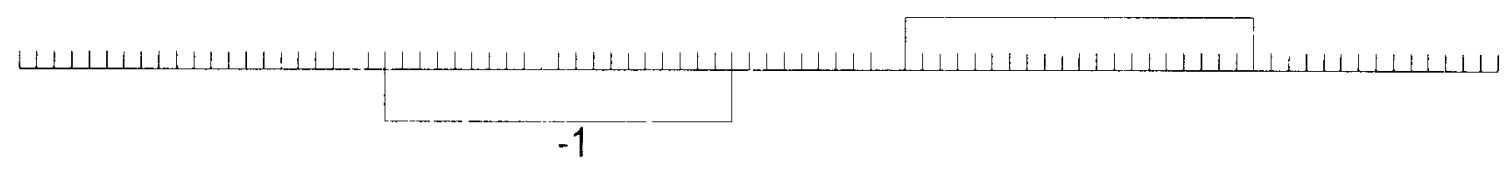

Figure $3 \mathrm{~A}$

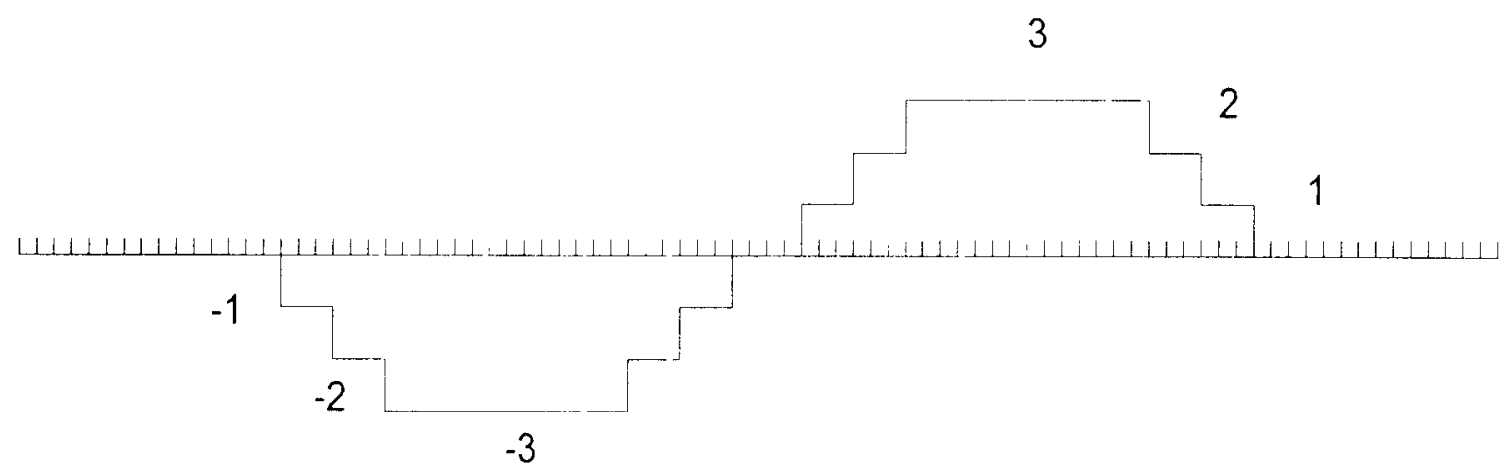

Figure $3 \mathrm{~B}$

Composite Line Current for ThreePower Supplies

Phase Back of $0,18, \& 36$ degrees.

Figure 3 


\section{Appendix 1}

\section{Power Balance/Fundamental Current}

For a six pulse bridge connection:

$$
\begin{aligned}
& \mathrm{V}_{\mathrm{dc}}=\sqrt{2} \mathrm{~V}_{\mathrm{LL}}\left(\frac{6}{\pi}\right) \operatorname{Sin}\left(\frac{\pi}{6}\right) \operatorname{Cos} \alpha \\
& \mathrm{V}_{\mathrm{dc}}=1.35 \mathrm{~V}_{\mathrm{LL}} \operatorname{Cos} \alpha
\end{aligned}
$$

Where $\alpha$ is the phase-back angle

$$
P_{d c}=I_{d c} V_{d c}=\sqrt{3} V_{L L} I_{f u n} \operatorname{Cos} \theta
$$

Where $\mathrm{I}_{\text {fun }}$ is the fundamental component of the AC line current.

$\theta$ is the angle between $I_{f u n}$ and $V_{I I}$. In this case $\alpha=\theta$

$$
\begin{gathered}
\mathrm{V}_{1}=\mathrm{V}_{2}=\frac{1}{1.35}=0.7407 \\
\mathrm{P}_{1}=\mathrm{P}_{\mathrm{dc}}=1 \\
\mathrm{P}_{\mathrm{ac}}=\mathrm{P}_{\mathrm{dc}} \quad \mathrm{P}_{2}=\mathrm{V}_{\mathrm{dc}} \mathrm{I}_{\mathrm{dc}}=0.866 \\
\mathrm{I}_{\text {fun } 1}=\frac{P 1}{\sqrt{3} V_{L L} \operatorname{Cos} \theta}=\frac{1}{\sqrt{3}(0.7407) 1}=0.7797 \\
\mathrm{I}_{\text {fun } 2}=\frac{P_{2}}{\sqrt{3} V_{L L} \operatorname{Cos} \theta}=\frac{866}{\sqrt{3}(0.7407) \operatorname{Cos} 30}=0.7797 \\
\mathrm{I}_{\text {fun }} \text { in }=\mathrm{I}_{\text {fun } 1}+\mathrm{I}_{\text {fun } 2}=0.7797+0.7797 \nless-30 \\
=1.506 \nless-15
\end{gathered}
$$




\section{Appendix 2}

\section{Harmonic Calculations}

Period $=2 \pi \quad f(x)=\frac{a_{0}}{2}+\sum_{n=1}^{\infty}(\operatorname{an} \operatorname{Cos} n \omega t+b n \operatorname{Sin} n \omega t)$

Fourier Analysis of:

Fig. $2 \Lambda$

$$
\begin{aligned}
& \text { an }=0 \text { (odd function) } \\
& b n=\frac{2}{\pi} \int_{0}^{\pi} f(w t) \operatorname{Sin} \frac{n \pi w t}{\pi} d w t \\
& \text { bn }=\frac{2}{\pi \mathrm{n}}\left[\left[_{\frac{\pi}{6}}^{\frac{5 \pi}{6}}-\operatorname{Cosn} \mathrm{wt}\right]=\frac{2}{\pi \mathrm{n}}\left[-\operatorname{Cos} \mathrm{n} \frac{5 \pi}{6}+\operatorname{Cos} \mathrm{n} \frac{\pi}{6}\right]\right.
\end{aligned}
$$

For rms values

$$
\text { bn }=\frac{2}{\pi n \sqrt{2}}\left[-\operatorname{Cos} \frac{5 \pi n}{6}+\operatorname{Cos} n \frac{\pi}{6}\right]
$$

\begin{tabular}{l|l}
$\mathrm{n}$ & bn rms \\
\hline 1 & 0.7797 \\
5 & -0.1559
\end{tabular}

Figure 2C

$$
\mathrm{bn}=\frac{2}{\pi \mathrm{n}}\left[\left[_{\frac{\pi}{12}}^{\frac{3 \pi}{12}}-\operatorname{Cos} \mathrm{n} \mathrm{wt}\right]+\left[_{\frac{3 \pi}{12}}^{\frac{3 \pi}{4}}-2 \operatorname{Cos} \mathrm{n} \mathrm{wt}\right]+\left[\left[_{\frac{3 \pi}{4}}^{\frac{11 \pi}{12}}-\operatorname{Cos} \mathrm{n} \mathrm{t}\right]\right]\right.
$$




$$
b n=\frac{2}{\pi n}\left[-\operatorname{Cos} \frac{\pi}{4} n+\operatorname{Cos} \frac{\pi}{12} n-2 \operatorname{Cos} \frac{3 \pi}{4} n+2 \operatorname{Cos} \frac{\pi}{4} n-\operatorname{Cos} \frac{11 \pi}{12} n+\operatorname{Cos} \frac{3 \pi}{4} n\right]
$$

$$
\text { bn } \mathrm{rms}=\frac{2}{\pi n \sqrt{2}}\left[-\operatorname{Cos} n \frac{\pi}{4} n+\operatorname{Cos} \frac{\pi}{12} n-2 \operatorname{Cos} \frac{3 \pi}{4} n+2 \operatorname{Cos} \frac{\pi}{4} n-\operatorname{Cos} \frac{11 \pi}{12} n+\operatorname{Cos} \frac{3 \pi}{4} n\right]
$$

Figure 3B

\begin{tabular}{l|l}
$\mathrm{n}$ & bn rms \\
\hline 1 & 1.506 \\
5 & .0807
\end{tabular}

$$
\text { bn rms }=\frac{2}{\pi n \sqrt{2}}\left[-\cos n \frac{\pi}{6}+\cos n \frac{\pi}{15}-2 \cos n \frac{4 \pi}{15}+2 \cos n \frac{\pi}{6}-3 \cos n \frac{11 \pi}{15}+3 \cos n \frac{4 \pi}{15}-2 \cos n \frac{5 \pi}{6}+2 \cos n \frac{11 \pi}{15}-\cos n \frac{14 \pi}{15}+\cos n \frac{5 \pi}{6}\right]
$$

\begin{tabular}{l|l}
$\mathrm{n}$ & bn rms \\
\hline 1 & 2.263 \\
5 & 0.156
\end{tabular}

\section{Phasor Analysis}

Sumation of $2 \mathrm{~A}+2 \mathrm{~A} \varangle 30$ (See Table I) corresponds to Fig. $2 \mathrm{C}$

$$
\begin{aligned}
& I_{1}=.7797+.7797 \varangle 30 \\
& I_{1}=.7797+.67524+j 0.38985 \\
& =1.4549+j 0.38985 \\
& \left|I_{1}\right|=1.506 \\
& I_{5}=.1559+.1559 \varangle 150^{\circ} \\
& \left|I_{5}\right|=.0807
\end{aligned}
$$

Sumation of $2 \mathrm{~A}+2 \mathrm{~A} \varangle 18^{\circ}+2 \mathrm{~A} \varangle 36^{\circ}$ (See Table I) corresponds to Fig. 3B

$$
\begin{aligned}
& I_{1}=.7797+.7797 \varangle 18^{\circ}+.7797 \varangle 36^{\circ} \\
& \quad=.7797+.7415+\mathrm{j} 0.24094+.6308+\mathrm{j} 0.4583 \\
& \quad=2.152+\mathrm{j} 0.69924 \\
& \left|I_{1}\right|=2.263 \\
& I_{5}=.1559+.1559 \varangle 90+.1559 \varangle 180 \\
& \left|I_{5}\right|=.156
\end{aligned}
$$




\section{Appendix 3}

\section{Harmonic Distortion}

Rms Value of Figure 2A

$\mathrm{Irms}=\sqrt{\frac{2}{2 \pi} \int_{0}^{\frac{2 \pi}{3}} I^{2} \max d w t}=\sqrt{\frac{1}{\pi}\left(1^{2} \frac{2 \pi}{3}\right)}=\sqrt{\frac{2}{3}}=0.8165$

Rms Value of Figure 2C

$\mathrm{I} \mathrm{rms}=\sqrt{\frac{2}{2 \pi}\left[2 \int_{0}^{\frac{\pi}{6}} 1^{2} \mathrm{dwt}+\int_{0}^{\frac{\pi}{2}} 2^{2} \mathrm{dwt}\right.}=\sqrt{\frac{1}{\pi}\left(\frac{2 \pi}{6}+\frac{4 \pi}{2}\right)}=1.5275$

Rms Value of Figure 3B

$I \mathrm{rms}=\sqrt{\frac{2}{2 \pi}\left[2 \int_{0}^{\frac{\pi}{10}} 1^{2} \mathrm{dwt}+2 \int_{0}^{\frac{\pi}{10}} 2^{2} \mathrm{dwt}+\int_{0}^{\frac{7 \pi}{15}} 3^{2} \mathrm{dwt}\right.}=\sqrt{\frac{1}{\pi}\left(\frac{2 \pi}{10}+\frac{8 \pi}{10}+\frac{63 \pi}{15}\right)}=2.2804$

Harmonic Distortion:

Id = Total Harmonic Distortion rms

$$
\begin{array}{cc}
\mathrm{I} \mathrm{rms}=\sqrt{\mathrm{I}_{\text {fun }}^{2}+\mathrm{I}_{\mathrm{D}}^{2}} & \sqrt{\sum_{\mathrm{n}=2}^{\infty} \mathrm{I}_{\mathrm{n}}^{2}}=\mathrm{I}_{\mathrm{D}} \\
\mathrm{I}_{\mathrm{D}}^{2}=\mathrm{I}_{\mathrm{rms}}^{2}-\mathrm{I}_{\text {fun }}^{2} & \sqrt{\sum_{\mathrm{n}=1}^{\infty} \mathrm{I}_{\mathrm{n}}^{2}}=\mathrm{I}_{\mathrm{rms}}
\end{array}
$$

Figure 3B

$\mathrm{Id}^{2}=(2.2804)^{2}-(2.263)^{2}=0.07883$

Id $=0.2807$

$I_{\mathrm{THD}}=\frac{\mathrm{I}_{\mathrm{D}} \times 100}{\mathrm{I}_{\text {fun }}}=\frac{0.2807 \times 100}{2.263}=12.4 \%$ 